\title{
Two-Color Radiation Generated in a Seeded Free-Electron Laser with Two Electron Beams
}

\author{
A. Petralia, ${ }_{1}^{1}$ M. P. Anania, ${ }^{2}$ M. Artioli, ${ }^{3}$ A. Bacci, ${ }^{4}$ M. Bellaveglia, ${ }^{2}$ M. Carpanese, ${ }^{1}$ E. Chiadroni, ${ }^{2}$ A. Cianchi, ${ }^{5}$ F. Ciocci,${ }^{1}$ \\ G. Dattoli, ${ }^{1}$ D. Di Giovenale, ${ }^{2}$ E. Di Palma, ${ }^{1}$ G. P. Di Pirro, ${ }^{2}$ M. Ferrario, ${ }^{2}$ L. Giannessi, ${ }^{1}$ L. Innocenti, ${ }^{5}$ A. Mostacci, ${ }^{6}$ \\ V. Petrillo, ${ }^{4,7}$ R. Pompili, ${ }^{2}$ J. V. Rau, ${ }^{8}$ C. Ronsivalle, ${ }^{1}$ A. R. Rossi, ${ }^{4}$ E. Sabia, ${ }^{1}$ V. Shpakov, ${ }^{2}$ C. Vaccarezza, ${ }^{2}$ and F. Villa ${ }^{2}$ \\ ${ }^{1}$ ENEA Centro Ricerche Frascati, Via Enrico Fermi 45, IT 00044 Frascati, Roma, Italy \\ ${ }^{2}$ INFN Laboratori Nazionali di Frascati, Via Enrico Fermi 44, 00044 Frascati, Roma, Italy \\ ${ }^{3}$ ENEA Centro Ricerche Bologna, Via Martiri Monte Sole 4, 40129 Bologna, Italy \\ ${ }^{4}$ INFN-Sezione di Milano, Via Celoria 16, 20133 Milano, Italy \\ ${ }^{5}$ Università Tor Vergata di Roma, Via della Ricerca Scientifica 1, 00133 Roma, Italy \\ ${ }^{6}$ Università La Sapienza di Roma, Via Antonio Scarpa 24, 00133 Roma, Italy \\ ${ }^{7}$ Università degli Studi di Milano, Via Celoria 16, 20133 Milano, Italy \\ ${ }^{8}$ ISM-CNR, Via del Fosso del Cavaliere 100, 00133 Roma, Italy
}

(Received 13 March 2015; published 30 June 2015)

\begin{abstract}
We present the experimental evidence of the generation of coherent and statistically stable two-color free-electron laser radiation obtained by seeding an electron beam double peaked in energy with a laser pulse single spiked in frequency. The radiation presents two neat spectral lines, with time delay, frequency separation, and relative intensity that can be accurately controlled. The analysis of the emitted radiation shows a temporal coherence and a shot-to-shot regularity in frequency significantly enhanced with respect to the self-amplified spontaneous emission.
\end{abstract}

DOI: 10.1103/PhysRevLett.115.014801

PACS numbers: 41.60.Cr, 41.50.+h, 42.55.Vc

Two-color radiation enables a wide number of new experiments, ranging from time-resolved analysis of the atomic, surface, and plasma dynamics to the imaging of biomedical samples and molecules. Studies on dual frequency production on free-electron lasers (FELs) have been recently carried on with different methods [1-5] in various frequency regimes, while, at the same time, several promising theoretical proposals have been investigated, aimed at generating two-color FEL emission in the x-ray wavelength range [6-9]. A particularly powerful method for producing two-color radiation is the double electron bunch operation that has been implemented and demonstrated in the visible $[2,10,11]$ and in the $\mathrm{x}$-ray [5] domains, in this latter case with applications on experiments with external users. This scheme is based on two closely spaced electron beamlets generated at the cathode and accelerated off crest along the linac to two different energies. The bunch train, driven in the undulator, radiates two distinct self-amplified spontaneous emission (SASE) pulses, whose relative time delay and wavelength difference can be tuned independently by changing the extraction conditions from the linac. The particular advantage of this approach is the large spectral separation, up to $1-2 \%$ [10], that can be achieved and the possibility of using the entire undulator length on both colors, thus allowing applications requiring high-intensity radiation or exploiting the activation of self-seeding processes. In all these previous experiments, the emission starts from noise and, whereas the two colors can be single spiked, the radiation is affected by random shot-to-shot fluctuations both in the time and frequency domains and presents, therefore, poor longitudinal coherence. The degree of coherence can be improved by seeding the radiation with a temporally coherent external field. This technique covers directly all the radiation frequencies where coherent sources are available. In particular, atomic lasers can be used to seed radiation in their range of operation. The use of the laser harmonics extends this method to ultraviolet [12], while the combination of high-gain harmonics generation (HGHG) with multistage cascade schemes permits to reach, at the status of the art, the water window [13].

In this Letter, we present an experimental demonstration of the operation with a seed, single spiked in frequency and time, applied to an electron beam with two peaks centered at two slightly different energies. The radiation presents two distinct colors occurring sequentially in time, with time delay, frequency separation, and relative intensity that can be controlled by moving the electron beam parameters. The study of the statistics of the emission shows the temporal coherence and regularity in frequency much enhanced with respect to the SASE case.

The experiment was performed at the SPARC_LAB facility [14] on the seeded SPARC FEL $[12,15,16]$. The double peak structure in the electron distribution was generated by illuminating the photocathode with a comb laser consisting of two pulses hundreds fs long separated by 4 ps [17]. The emitted ps-spaced electron beam was then injected in the first of the three $S$ band sections of the linac close to the zero-crossing radio frequency (rf) field phase, 
TABLE I. Electron beam parameters relevant to the total beam and to the two beamlets $a$ and $b$.

\begin{tabular}{lccc}
\hline \hline & Total & Beamlet $a$ & Beamlet $b$ \\
\hline Mean energy (MeV) & 94.8 & 95.3 & 94.3 \\
Charge (pC) & 100 & 50 & 50 \\
Peak current (A) & 310 & 110 & 200 \\
rms pulse duration (fs) & 170 & 190 & 100 \\
Emittance(mm-mrad) & 2.1 & & \\
Energy spread (MeV) & 0.5 & 0.13 & 0.1 \\
\hline \hline
\end{tabular}

where it is compressed by velocity bunching [18-20]. The $e$ beam was then extracted at the maximum compression, where the two beamlets are overlapped in time and separated in energy. The linac rf phases of the last two sections were tuned in order to accelerate the beam and optimize the energy spread. The final mean energy of the beam was $E=95 \mathrm{MeV}$, allowing FEL emission at $800 \mathrm{~nm}$, an optimal condition for the availability of the seed and for the radiation diagnostics. The electron beam parameters in the operational conditions are listed in Table I. The experimental longitudinal phase space, Fig. 1, shows two electron beamlets with a rms energy spread of $0.1 \mathrm{MeV}$ each, separated by $1 \mathrm{MeV}$ and with total rms temporal duration of $170 \mathrm{fs}$.

The electron beam was then injected in the undulator system, consisting of six modules of 75 periods each, with $\lambda_{u}=2.8 \mathrm{~cm} \mathrm{[21],} \mathrm{tuned} \mathrm{at} \mathrm{the} \mathrm{resonant} \mathrm{wavelength}$ $\lambda=\left(\lambda_{u} / 2 \gamma^{2}\right)\left(1+K^{2} / 2\right)=800 \mathrm{~nm}$, where $K$ is the undulator deflecting parameter and $\gamma$ is the average value of the electron Lorentz factor. The optimum matching condition of

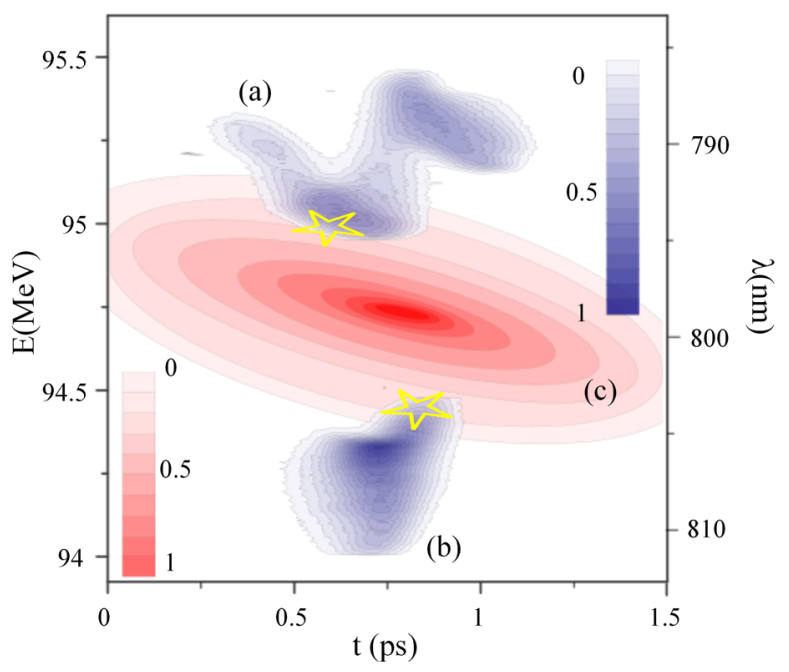

FIG. 1 (color online). The electron phase space composed of two beamlets $a$ and $b$ compared to the laser chirped distribution (c). The higher-energy beamlet (a) appears to be fragmented in two parts, due to the space charge effects. Only the zone seeded by the laser takes part to the radiation process. Stars show the regions where the FEL emission is supposed to take place according to the experimental results.
TABLE II. Seed laser parameters of the experimental setup.

\begin{tabular}{lc}
\hline \hline Central wavelength & $800 \mathrm{~nm}$ \\
Spectral bandwidth (FWHM) & $7 \mathrm{~nm}$ \\
Pulse duration (FWHM) & $800 \mathrm{fs}$ \\
Total pulse energy & $810 \mathrm{~nJ}$ \\
\hline \hline
\end{tabular}

the electron beam to the undulator was found by using the average energy and the average projected values for the emittance and Twiss parameter [22], calculated after measuring them separately for the two bunches in a dispersive arm. This procedure allows us to balance the effect of the matching on the radiation's gain process for the two beamlets.

The one-dimensional Pierce parameter [23] for each beamlet can be estimated as $\rho=4 \times 10^{-3}$, corresponding to a cooperation length of $L_{c}=9 \mu \mathrm{m}$.

SASE radiation was observed. The significant compression of the electron beam and the relatively large value of the current lead the system in the single-spike radiation condition $L_{b} \lesssim 2 \pi L_{c}$, where $L_{b} \approx 45 \mu \mathrm{m}$ is the electron beam width. A fine-tuning of the last undulator module (by varying the gap of 1\%) allowed us to maximize the output power.

The seed-laser system [24] consists of a Ti:sapphire regenerative amplifier (Legend HFE, by Coherent), driven by the same oscillator as the photocathode laser and it has been set to deliver up to $450 \mu \mathrm{J}$ at $\lambda_{0}=800 \mathrm{~nm}$. The seed-laser pulse parameters of the experimental setup are reported in Table II.

A small linear chirp (see Fig. 1) has been introduced to lengthen the seed pulse, ensuring superposition with both the electron bunches and increasing the tolerance for the synchronization with the beam. The laser profile is assumed Gaussian in both time and frequency domains, with a distribution

$$
E(\omega, t) \propto e^{-\left[\left(\left(t-t_{0}\right)^{2} / \sigma_{t}^{2}\right)+\left[2 \alpha\left(t-t_{0}\right)+\left(\omega-\omega_{0}\right)\right]^{2} \sigma_{t}^{2}\right]},
$$

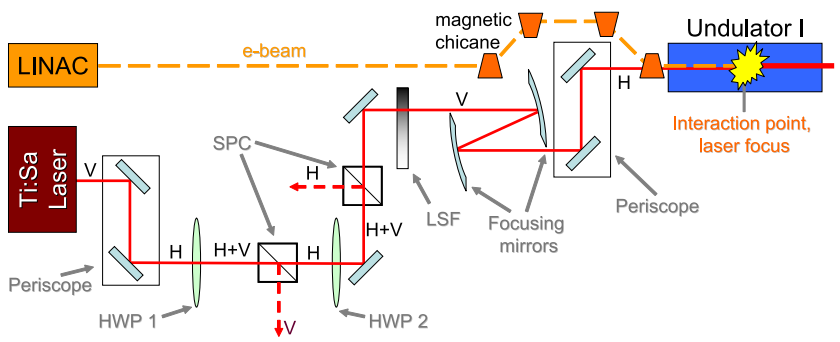

FIG. 2 (color online). Optical transport setup for the seeding laser. The laser intensity on the horizontal $(\mathrm{H})$ polarization is attenuated with coupling a half-wave plate (HWP) and a splitting polarizer cube (SPC). The plate rotates the polarization and the splitting cube selects only the useful component for the interaction with the electron beam. In addition, a linear step filter (LSF) allows more attenuation steps. Finally, two spherical mirrors focus the laser, with an expected size $\sigma \approx 600 \mu \mathrm{m}$, at the center of the first undulator module, where it interacts with the electron beam. With a Rayleigh length $z_{r}=0.5 \mathrm{~m}$, the interaction in the next undulator modules can be neglected. 


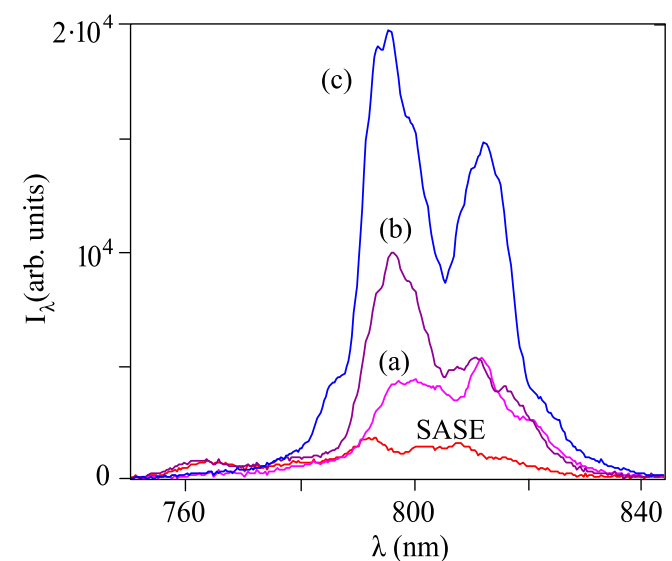

FIG. 3 (color online). Spectral intensity $I_{\lambda}$ versus wavelength acquired with the fiber spectrometer. The average spectral intensity $\left\langle I_{\lambda}\right\rangle$ from 100 shots is shown. Seeded FEL spectra are compared for different values of the total seed-laser energy per pulse at the interaction point: (a) $E_{0}=15 \mathrm{~nJ}$, (b) $E_{0}=125 \mathrm{~nJ}$, and (c) $E_{0}=810 \mathrm{~nJ}$. The SASE spectrum (red line) is also pointed out.

where $\alpha$ is the chirp parameter and $\sigma_{t}$ the rms temporal width.

The seed-laser signal is attenuated before injection in the undulator with the scheme shown in Fig. 2. Attenuation is realized in two steps, where a half-wave plate changes the polarization of the light, and a polarizing splitting cube selects the component with polarization parallel to the oscillation plane of the electrons in the linear undulator. The two steps allow us to reduce the laser pulse energy and to remotely tune the energy in the range from $810 \mathrm{~nJ}$ to a few $\mathrm{nJ}$, by rotating the second wave plate.

A single laser pulse is therefore used to seed simultaneously the two electron bunches. The small energy chirp on the seed pulse probably contributes to produce a temporal separation of the two FEL pulses. The spectral region of the laser, actually acting as seed, is the portion on the wings of the Gaussian distribution (see the overlapping region in Fig. 1), that can be estimated by integrating the seed energy distribution [see Eq. (1)] both in time and wavelength, choosing the temporal length and spectral bandwidth as intervals of integration for each pulse. This evaluation gives an effective seed energy in the range $1-10 \mathrm{~nJ}$.

The undulator was tuned at $800 \mathrm{~nm}$, the central wavelength of the seed laser. In this way, the two FEL pulses generated in the undulator have their own resonant

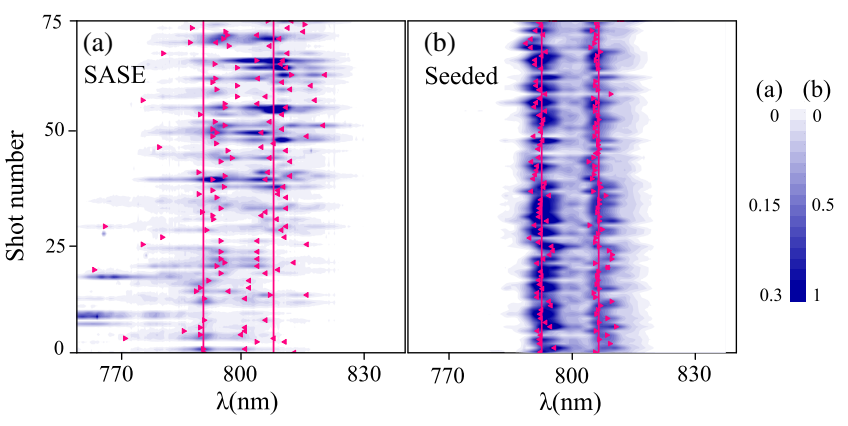

FIG. 4 (color online). Spectral intensity vs wavelength. Sequence of shots without (a) and with (b) seed. Pink lines, average wavelength $\left\langle\lambda_{1,2}\right\rangle$; pink triangles, peak values of each shot.

wavelengths, $\lambda_{1,2}=\left(\lambda_{u} / 2 \gamma_{1,2}^{2}\right)\left(1+K^{2} / 2\right)$, whose relative difference $\delta \lambda / \lambda=2 \delta E / E$ depends on the energy separation of the two electron bunches. Since $\delta E \approx 1 \mathrm{MeV}$, the expected wavelength separation $\delta \lambda$ is $16.5 \mathrm{~nm}$.

A fiber spectrometer and a Grenouille frequencyresolved optical gating (FROG) [25] are used as diagnostics for the direct detection of the light spectrum and time distribution. The FEL radiation is split in such a way (5\% to the spectrometer, 95\% to the FROG) that both devices acquire at the same time images of the same shot.

Figure 3 presents spectra (average on 100 shots) of the two-color radiation for different values of the seed-laser energy, starting from the pure SASE mode. The radiation shows two distinct spectral lines, whose intensity grows with increasing seed energy. In both SASE and seeded mode, wavelength separations $\delta \lambda_{\mathrm{SASE}}=15.9 \mathrm{~nm}$ and $\delta \lambda_{s}=14.7 \mathrm{~nm}$ are in agreement with the value deduced from the electron energy gap. An increase in intensity by a factor 10 and an enhancement in the visibility of the two colors from $30 \%$ to $50 \%$ between the SASE and the optimized seeded case were observed.

Figure 4 shows the complete sequences of 100 shots measured on the spectrometer for SASE and seeded mode. Some statistical spectral parameters of the radiation are presented in Table III. In SASE mode, only a fraction of $66 \%$ of the shots has a double-peaked configuration, while the other ones have SASE or single-spike structure. In the seeded mode, instead, the double-peaked spectra are $99 \%$ of the acquired shots and the average wavelength values $\left\langle\lambda_{1,2}\right\rangle$ are in agreement with the data of $\lambda_{1, s}$ and $\lambda_{2, s}$ read in the average spectrum. The measured bandwidths in the seeded cases are less than one half compared to the ones in

TABLE III. Statistical properties for the two central wavelength $\left(\lambda_{1}\right.$ and $\left.\lambda_{2}\right)$, wavelength separation $\left(\lambda_{1}-\lambda_{2}\right)$ and bandwidth (bw) for the two radiation pulses. All quantities are expressed in $\mathrm{nm}$.

\begin{tabular}{lccccc}
\hline \hline & $\left\langle\lambda_{1}\right\rangle$ & $\left\langle\lambda_{2}\right\rangle$ & $\left\langle\lambda_{1}-\lambda_{2}\right\rangle$ & $\left\langle b w_{1}\right\rangle$ & $\left\langle b w_{2}\right\rangle$ \\
\hline SASE & $790.8 \pm 10$ & $808 \pm 6.3$ & $17.4 \pm 10.6$ & $7.2 \pm 1.7$ & $7.9 \pm 1.5$ \\
Seeded mode & $792.5 \pm 1.5$ & $806.6 \pm 1.8$ & $15.1 \pm 1.2$ & $3.1 \pm 0.5$ & $2.7 \pm 0.6$ \\
\hline \hline
\end{tabular}




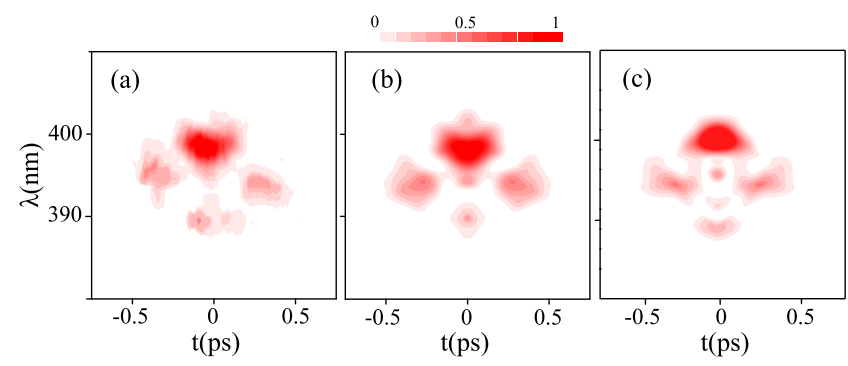

FIG. 5 (color online). FROG traces (a) raw, (b) retrieved and (c) simulated with GENESIS 1.3 using a two energy electron beam with parameters similar to Table I.

the SASE spectra. The relative fluctuations in average wavelength, spectral separation and bandwidth (bw) in the SASE mode are, respectively, $\Delta \lambda_{1,2} /\left\langle\lambda_{1,2}\right\rangle \approx 0.013(0.007)$; $\Delta \delta \lambda /\langle\delta \lambda\rangle \approx 0.47 ; \Delta b w_{1,2} /\left\langle b w_{1,2}\right\rangle=0.23(0.19)$, where $\Delta$ stands for the standard deviation of the distributions; and $\delta \lambda=\lambda_{2}-\lambda_{1}$. The seeded case presents a much more stable and coherent configuration with, respectively, $\Delta \lambda_{1,2} /\left\langle\lambda_{1,2}\right\rangle \approx 0.0019(0.0022), \quad \Delta \delta \lambda /\langle\delta \lambda\rangle \approx 0.08, \quad$ and $\Delta b w_{1,2} /\left\langle b w_{1,2}\right\rangle=0.16(0.22)$. Also, the radiation intensity fluctuations are reduced from $\Delta I_{\lambda} / I_{\lambda} \approx 0.53$ of the SASE to $\Delta I_{\lambda} / I_{\lambda} \approx 0.18$ of the seeded mode.

Measurements done with the FROG permitted us to acquire both spectral and temporal profiles. However, the FROG technique requires a radiation energy level larger than a threshold of about $1 \mu \mathrm{J}$. In the SASE case, the emission was marginally weaker than this value, and only few useful FROG traces were generated, not permitting a statistical analysis. In the seeded regime, instead, 95 shots over 100 were widely above the threshold. The comparison between the two cases allowed us to quote the seeded emission energy at the value of about $10 \mu \mathrm{J}$.

An example of a FROG trace is shown in Fig. 5. Since the radiation is composed by two pulses spectrally and temporally separated, and coming from two electron beamlets propagating in the undulator with different trajectories, the two FEL pulses may enter the FROG with slightly different angles. This results in temporally asymmetric traces in a second-order FROG. For this reason, the data were selected on the basis of temporal symmetry by computing the cross correlation between the right and the left part of the raw trace $A$ averaged on frequency and normalized to intensity,

$$
\Gamma=\frac{\int d \omega \int A(\omega, t) A(\omega,-t) d t}{\int d \omega \int A^{2}(\omega, t) d t} .
$$

The factor $\Gamma$ is 0 for noise and 1 for completely symmetrical traces. Only 48 shots over 95 were retained by assuming $\Gamma>0.5$ as a threshold. The sequence in the time domain of the seeded radiation, as measured with the FROG, is presented in Fig. 6.

The measured temporal profile of the FEL pulses shows systematically a double-peak structure. The mean

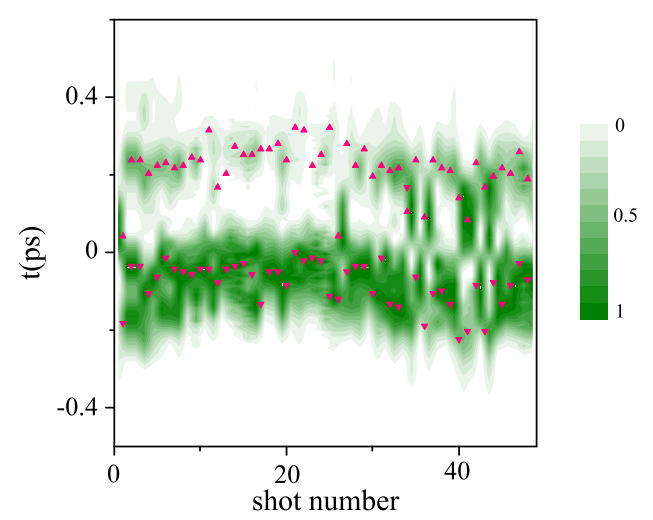

FIG. 6 (color online). Sequence of the selected FROG time distributions. The pink triangles are the peaks of the distributions.

separation in time over the acquired number of shots is $\langle\delta t\rangle=294 \pm 71 \mathrm{fs}$, a value compatible with the temporal distance $\delta \tau=230$ fs between the positions $\tau_{1,2}$ of the two beamlets evaluated by averaging on the relative current distribution $\tau_{1,2}=\int t I_{1,2}(t) d t / \int I_{1,2}(t) d t$ (see Fig. 7). Even if the two electron bunches are overlapped in time, the regions in the beamlets where the radiation process actually occurs seem not exactly superimposed. This separation could be justified by the energy chirp on the seed laser favoring the laser process in different regions of the higher and lower energy beams, respectively.

The result of a start-to-end simulation done with T-STEP [26] and GENESIS 1.3 [27] is presented in Fig. 5(c). The analysis of the experimental data has been also performed with a semianalytical code based on equations deduced by employing the method of the FEL approximants [28]. This procedure [29] allows the treatment of the two-color

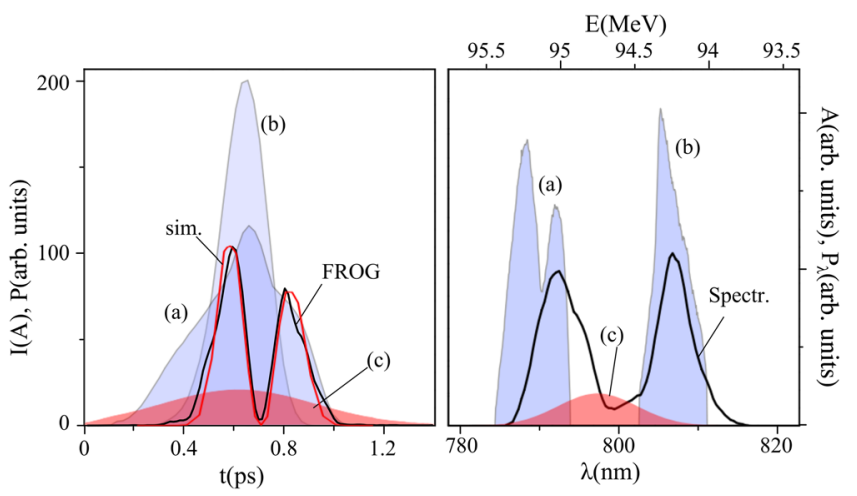

FIG. 7 (color online). Comparison between the position of the experimental FEL pulses (blue continuous line) and the two electron beamlets (a) and (b) in time (left) and energy (right) distribution. The seed-laser profile (c) is shown (not in scale). All ordinate scales, showing the intensity, are in arbitrary units except for the current profile $I(A)$ given in ampere. The experimental FEL pulse was measured with FROG and a spectrometer. The magenta dashed curve is the semianalytical simulation made with the parameters of Tables I and II. 
operation with the inclusion of pulse propagation effects as well as other beam characteristics (emittance, energy spread, and bunch shape) and permits us to explore, in real time, a wide range of experimental conditions. The result obtained with the parameters of Tables I and II is reported in Fig. 7.

In conclusion, we have presented the experimental evidence of the generation of coherent and statistically stable free-electron laser two-color radiation obtained by seeding electron beams double peaked in energy with laser pulses single spiked in time and frequency. This method produces high-quality light and could be extrapolated toward higher frequencies using high-gain harmonic generation or self-seeding techniques.

[1] A. A. Lutman, R. Coffee, Y. Ding, Z. Huang, J. Krzywinski, T. Maxwell, M. Messerschmidt, and H.-D. Nuhn, Phys. Rev. Lett. 110, 134801 (2013).

[2] V. Petrillo et al., Phys. Rev. Lett. 111, 114802 (2013).

[3] E. Allaria et al., Nat. Commun. 4, 2476 (2013).

[4] B. Mahieu et al., Opt. Express 21, 22728 (2013); G. De Ninno, B. Mahieu, E. Allaria, L. Giannessi, and S. Spampinati, Phys. Rev. Lett. 110, 064801 (2013).

[5] A. Marinelli et al., Nat. Commun. 6, 6369 (2015); T. Hara et al., Nat. Commun. 4, 2919 (2013).

[6] H. P. Freund and P. G. O’Shea, Phys. Rev. Lett. 84, 2861 (2000).

[7] N. R. Thompson and B. W. J. McNeil, Phys. Rev. Lett. 100, 203901 (2008).

[8] D. Xiang, Z. Huang, and G. Stupakov, Phys. Rev. ST Accel. Beams 12, 060701 (2009).

[9] A. Zholents and G. Penn, Nucl. Instrum. Methods Phys. Res., Sect. A 612, 254 (2010).
[10] C. Ronsivalle et al., New J. Phys. 16, 033018 (2014).

[11] E. Chiadroni et al., Phys. Procedia 52, 27 (2014).

[12] L. Giannessi et al., Phys. Rev. Lett. 108, 164801 (2012).

[13] E. Allaria et al., Nat. Photonics 7, 913 (2013).

[14] M. Ferrario et al., Nucl. Instrum. Methods Phys. Res., Sect. A 309, 183 (2013).

[15] L. Giannessi et al., Phys. Rev. Lett. 110, 044801 (2013).

[16] M. Labat et al., Phys. Rev. Lett. 107, 224801 (2011).

[17] F. Villa, S. Cialdi, M. P. Anania, G. Gatti, F. Giorgianni, and R. Pompili, Nucl. Instrum. Methods Phys. Res., Sect. A 740, 188 (2014).

[18] M. Ferrario et al., Phys. Rev. Lett. 104, 054801 (2010).

[19] L. Giannessi et al., Phys. Rev. Lett. 106, 144801 (2011).

[20] G. Marcus et al., Appl. Phys. Lett. 101, 134102 (2012).

[21] M. Quattromini, M. Artioli, E. DiPalma, A. Petralia, and L. Giannessi, Phys. Rev. ST Accel. Beams 15, 080704 (2012).

[22] G. Dattoli, E. Sabia, C. Ronsivalle, M. Del Franco, and A. Petralia, Nucl. Instrum. Methods Phys. Res., Sect. A 671, 51 (2012).

[23] R. Bonifacio, C. Pellegrini, and L. Narducci, Opt. Commun. 50, 373 (1984).

[24] C. Vicario et al., EUROFEL Report No. 2007-DS4-093.

[25] R. Trebino, Frequency-Resolved Optical Gating: The Measurement of Ultrashort Laser Pulses (Kluwer Academic, Norwell, MA, 2000).

[26] T-STEP is an upgraded version of Parmela; see, L. M. Young, PARMELA, Los Alamos National Laboratory Report No. LAUR-96-1835.

[27] S. Reiche, Nucl. Instrum. Methods Phys. Res., Sect. A 429, 243 (1999).

[28] G. Dattoli, L. Giannessi, A. Torre, and A. Segreto, J. Appl. Phys. 79, 6729 (1996).

[29] G. Dattoli, E. Sabia, P. L. Ottaviani, S. Pagnutti, and V. Petrillo, Phys. Rev. ST Accel. Beams 16, 030704 (2013). 